\title{
Helical Magnetic Fields from Sphaleron Decay and Baryogenesis
}

\author{
Craig J. Copi ${ }^{1}$, Francesc Ferrer ${ }^{1}$, Tanmay Vachaspati ${ }^{1,2}$ and Ana Achúcarro ${ }^{3,4}$ \\ ${ }^{1}$ CERCA, Department of Physics, Case Western Reserve University, \\ 10900 Euclid Avenue, Cleveland, OH 44106-7079, \\ ${ }^{2}$ Institute for Advanced Study, Princeton, NJ 08540 \\ ${ }^{3}$ Instituut-Lorentz for Theoretical Physics, Leiden, The Netherlands, \\ ${ }^{4}$ Department of Theoretical Physics, The University of the Basque Country UPV-EHU, 38940 Bilbao, Spain
}

\begin{abstract}
Many models of baryogenesis rely on anomalous particle physics processes to give baryon number violation. By numerically evolving the electroweak equations on a lattice, we show that baryogenesis in these models creates helical cosmic magnetic fields. After a transitory period, electroweak dynamics is found to conserve the Chern-Simons number and the total electromagnetic helicity. We argue that baryogenesis could lead to magnetic fields of nano-Gauss strength today on astrophysical length scales. In addition to being astrophysically relevant, such helical magnetic fields can provide an independent probe of baryogenesis and $\mathrm{CP}$ violation in particle physics.
\end{abstract}

Cosmic magnetic fields can arise from a number of early universe processes (e.g. [1, 2, 3, 4, 5, 6]). The detection of such magnetic fields would be an important step in our understanding of structure evolution, and the magnetic field distribution could be used as a probe of early universe cosmology. Most magnetic field generation mechanisms discussed so far, produce non-helical fields, and thus it is of particular interest that processes like electroweak baryogenesis imply magnetic fields that are helical [5, 6]. If helical primordial magnetic fields are observed, they would offer confirmation of cosmological baryogenesis, and the magnetic field properties could be turned into a detailed probe of particle physics and cosmology at the epoch of baryogenesis. In particular, the $\mathrm{CP}$ violation that leads to a universe filled with matter and no antimatter would be probed by cosmological observations.

These considerations have prompted us to study the production of magnetic fields during the decay of sphalerons [9, 10], a process that is key to electroweak baryogenesis. While electroweak baryogenesis is not successful in the minimal standard model, our study also applies to any extension of the standard model in which baryon number violation is mediated by sphaleronlike processes involving changes in the winding (ChernSimons number) of vacuum non-Abelian gauge field configurations.

To understand the connection between sphalerons and helical magnetic fields, it is simpler to think of "deformed sphalerons" where the gauge field configuration resembles that of electroweak strings [11]. The sphaleron can then be interpreted as linked loops of electroweak Z-string or a confined electroweak monopole-antimonopole pair [12, 13, 14, 15]. The linked loops of $\mathrm{Z}$ magnetic flux can then decay into linked electromagnetic flux as described in [6] and thus the resulting electromagnetic field carries magnetic helicity. If we think of the sphaleron in terms of the confined magnetic monopole pair, the string that confines them is twisted and this also leads to magnetic helicity. In [5, 6 ] these considerations indicated a remark- ably simple relationship between the cosmic magnetic helicity density and the cosmic baryon number density

$$
h=\frac{1}{V} \int_{V} d^{3} x \mathbf{A} \cdot \nabla \times \mathbf{A} \sim-\frac{n_{b}}{\alpha}
$$

where we consider some large spatial volume $V, \mathbf{A}$ is the electromagnetic vector potential, $n_{b}$ is the average baryon number density and $\alpha=1 / 137$ is the fine structure constant.

Our goal is to examine the heuristic arguments in [5, 6] by explicitly studying the decay of an electroweak sphaleron. (Recently, along similar lines, magnetic fields produced during preheating at the electroweak scale were studied in Ref. [7].) We will indeed find that helical magnetic fields are generated during sphaleron decay and the relation in Eq. (1) holds at the order of magnitude level. Our numerical results also show, somewhat unexpectedly, that the Chern-Simons number and the electromagnetic helicity are conserved after a transitory initial period (also see [8]). We also reconsider the net magnetic field generated during baryogenesis and find that the field strength is likely to be much larger than has been previously estimated.

We work with the bosonic sector of the electroweak Lagrangian

$$
\begin{aligned}
\mathcal{L} & =\left(D_{\mu} \Phi\right)^{\dagger} D^{\mu} \Phi-\frac{1}{4} B_{\mu \nu} B^{\mu \nu}-\frac{1}{4} \boldsymbol{W}_{\mu \nu} \cdot \boldsymbol{W}^{\mu \nu} \\
& -\lambda\left(|\Phi|^{2}-v^{2}\right)^{2}
\end{aligned}
$$

where

$$
D_{\mu} \Phi=\left(\partial_{\mu}-i \frac{g^{\prime}}{2} B_{\mu}-i \frac{g}{2} \boldsymbol{\tau} \cdot \boldsymbol{W}_{\mu}\right) \Phi
$$

The $S U(2)$ generators, $\tau^{a}$, are normalized by $\operatorname{Tr}\left(\tau^{a} \tau^{b}\right)=$ $2 \delta^{a b}$. The $U(1)$ (hypercharge) field strength is

$$
B_{\mu \nu}=\partial_{\mu} B_{\nu}-\partial_{\nu} B_{\mu},
$$

and the $S U(2)$ field strength is defined as

$$
W_{\mu \nu}^{a}=\partial_{\mu} W_{\nu}^{a}-\partial_{\nu} W_{\mu}^{a}+g \epsilon^{b c a} W_{\mu}^{b} W_{\nu}^{c}
$$


To study the magnetic field produced by a decaying sphaleron, we numerically set up a configuration like the electroweak sphaleron solution [9, 10, 17], i.e. a perturbed sphaleron, since the exact solution, even though it is unstable, will take a long time to decay numerically.

$$
\begin{gathered}
\Phi=v h(\xi) \mathbf{G}_{\Theta}\left(\begin{array}{c}
i x+y \\
-i z
\end{array}\right) \\
W_{i}^{a} \tau^{a}=-\frac{2 f(\xi)}{g r^{2}} \epsilon_{i c b} x_{b} \mathbf{G}_{\Theta} \tau^{c} \mathbf{G}_{\Theta}^{\dagger} \\
B_{i}=g^{\prime} v^{2} p(\xi)(-y, x, 0)
\end{gathered}
$$

where

$$
\mathbf{G}_{\Theta}(\vec{x})=\exp [i \Theta(r) \boldsymbol{\tau} \cdot \hat{\mathbf{x}} / 2]
$$

is an $S U(2)$ gauge transformation, with $\Theta(r)=$ $\pi(1-\exp (-r))$, ensuring that the gauge fields fall off fast enough away from the origin. The functions $f(\xi)$, $h(\xi)$ and $p(\xi)$ are profile functions and are taken to depend on the radial coordinate $\xi=g v r / \sqrt{2}$ alone.

In the true solution, the profile functions also depend on the angular coordinates and have to be determined numerically [17]. Since we want to start with a perturbed sphaleron we do not need the precise forms of $f$ and $h$, but we do need to meet the asymptotic properties satisfied by $f$ and $h: f, h \rightarrow 0$ as $r \rightarrow 0$, and $f, h \rightarrow$ 1 as $r \rightarrow \infty$. Following Klinkhamer and Manton [10], we choose for the profile functions their Ansatz, labeled $a$, with the length scales given by $\Xi=3.79$ and $\Omega=$ 1.90. We take for the initial $U(1)$ gauge field, Eq. (8), the small $g^{\prime}$ approximation [10]. The profile function $p$ can be found, in terms of $f$ and $h$, by solving the $U(1)$ equation of motion in the $S U(2)$ background.

The decay is studied by evolving the $S U(2) \times U(1)$ electroweak field equations using the standard Wilsonian approach for lattice gauge fields [18, 19, 20, 21, 22]. The temporal gauge, $W_{0}^{a}=B_{0}=0$, allows a simple identification of the canonical momentum, and we use it throughout in this work. We adopt Graham's implementation of the lattice equations [22]

$$
\begin{aligned}
\phi(t+)= & 2 \phi(t)-\phi(t-)+(\Delta t)^{2}\left[2 \lambda\left(v^{2}-\left|\phi^{p}\right|^{2}\right) \phi^{p}\right. \\
& \left.+\sum_{i=x, y, z} \frac{U_{i}^{p} \phi^{p+i}-2 \phi^{p}+U_{-i}^{p} \phi^{p-i}}{\Delta x^{2}}\right]
\end{aligned}
$$

where the gauge fields are on the links and are defined in terms of the $U_{j}^{p}$ matrices by

$$
U_{j}^{p}=\mathrm{e}^{i g^{\prime} B_{j}^{p} \Delta x / 2} \mathrm{e}^{i g \boldsymbol{W}_{j}^{p} \cdot \boldsymbol{\tau} \Delta x / 2} .
$$

Here $p$ labels the point in the lattice and $j$ the link emanating from point $p$. The $U$ matrix along a timelike link, $j=t$, is equal to the identity matrix in the temporal gauge. For the link from site $p$ in the negative $j$ direction, we take:

$$
U_{-j}^{p}=\left(U_{j}^{p-j}\right)^{\dagger}=\mathrm{e}^{-i g^{\prime} B_{j}^{p-j} \Delta x / 2} \mathrm{e}^{-i g \boldsymbol{W}_{j}^{p-j} \cdot \boldsymbol{\tau} \Delta x / 2} .
$$

The evolution of the gauge fields is given by

$$
\begin{aligned}
U_{j}^{p}(t+)= & \exp \left[\log U_{j}^{p}(t) U_{j}^{p \dagger}\left(t_{-}\right)\right. \\
& -\frac{\Delta t^{2}}{\Delta x^{2}} \sum_{j^{\prime} \neq j}\left(\log U_{\square j j^{\prime}}^{p}+\log U_{\square j-j^{\prime}}^{p}\right) \\
& \left.+\frac{1}{2} i \Delta t^{2} \Delta x\left(g^{\prime} J_{j}^{p}+g \boldsymbol{J}_{j}^{p} \cdot \boldsymbol{\tau}\right)\right] U_{j}^{p}
\end{aligned}
$$

Here, we define $U_{\square j j^{\prime}}^{p} \equiv U_{j}^{p} U_{j^{\prime}}^{p+j} U_{-j}^{p+j+j^{\prime}} U_{-j^{\prime}}^{p+j^{\prime}}$ for the plaquette and $J_{j}^{p} \equiv-g^{\prime} \operatorname{Im}\left(\phi^{p \dagger} U_{j}^{p} \phi^{p+j}\right) / \Delta x$ and $\boldsymbol{J}_{j}^{p} \equiv$ $-g^{\prime} \operatorname{Im}\left(\phi^{p \dagger} \tau U_{j}^{p} \phi^{p+j}\right) / \Delta x$ for the gauge currents. For further details, the reader is referred to [22]. In addition to these evolution equations we have implemented absorbing boundary conditions as described in [23] but extended to non-Abelian fields.

The evolution of Chern-Simons number is of particular interest to us since it is correlated with changes in the baryon number. It is given by

$$
\begin{aligned}
N_{C S}(t) & =\frac{N_{F}}{32 \pi^{2}} \epsilon^{i j k} \int d^{3} x\left[-g^{\prime 2} B_{i j} B_{k}\right. \\
& \left.+g^{2}\left(W_{i j}^{a} W_{k}^{a}-\frac{g}{3} \epsilon_{a b c} W_{i}^{a} W_{j}^{b} W_{k}^{c}\right)\right]
\end{aligned}
$$

where $N_{F}=3$ is the number of families and there is no surface term because our fields vanish at infinity.

In the electroweak model, the electromagnetic gauge field $A_{\mu}$ is defined in terms of the electroweak $W^{\mu}$ and $B^{\mu}$ gauge fields

$$
A_{\mu}=\sin \theta_{w} n^{a} W_{\mu}^{a}+\cos \theta_{w} B_{\mu}
$$

with $n^{a}=-\Phi^{\dagger} \tau^{a} \Phi / \Phi^{\dagger} \Phi$. However, there is a choice of definitions for the electromagnetic field strength. For example,

$$
A_{\mu \nu}=\sin \theta_{w} n^{a} W_{\mu \nu}^{a}+\cos \theta_{w} B_{\mu \nu}
$$

is the natural definition to calculate the energy density in the massless electromagnetic field. Yet this definition is not simply the curl of the gauge field in Eq. (14) because derivatives of $A_{\mu}$ also involve derivatives of $n^{a}$. Even at late times, when $D_{\mu} \Phi$ becomes small, such derivatives will in general contribute. The only clean resolution of this issue that we have found is to define the electromagnetic gauge field in unitary gauge and then define the electromagnetic field strength as the curl of the gauge potential as in the usual Maxwell theory. At early times, 
when $D_{\mu} \Phi$ is significant, $\boldsymbol{\nabla} \times \mathbf{A}$ does not coincide with $A_{i j}$ in Eq. (15), but they do coincide at late times. Then the helicity tells us something about the topology of the very same magnetic field lines that carry energy density.

To go into unitary gauge, we calculate $A_{\mu}$ at every time step only after applying the $\mathrm{SU}(2)$ gauge rotation

$$
g_{2}=\frac{1}{\sqrt{\Phi^{\dagger} \Phi}}\left(\begin{array}{cc}
\Phi_{2} & -\Phi_{1} \\
\Phi_{1}^{*} & \Phi_{2}^{*}
\end{array}\right)
$$

where $\Phi_{1}$ and $\Phi_{2}$ are the upper and lower components of $\Phi$. It is easily verified that $g_{2} \Phi=\sqrt{\Phi^{\dagger}} \Phi(0,1)^{T}, \operatorname{det} g_{2}=1$ and $g_{2}^{\dagger}=g_{2}^{-1}$. After the rotation, $n^{a}=(0,0,1)$, and when the rotation is applied to $W_{\mu}^{a}$ the inhomogeneous part of the gauge transformation contributes in a nontrivial way to $A_{\mu}$. At late times, we then find that $\epsilon^{i j k} A_{j k} / 2$ coincides with $(\boldsymbol{\nabla} \times \mathbf{A})^{i}$, and we can unambiguously keep track of the total electromagnetic helicity defined as $\mathcal{H}(t)=V h(t)$ (see Eq. (1)).

In Fig. 1 we show the time evolution of the ChernSimons number and the total electromagnetic helicity. We see changes in the Chern-Simons number as well as growth in the magnitude of the electromagnetic helicity, and then a period of conservation (time step 50 to 250). While electromagnetic helicity is known to be conserved in a conducting plasma, its conservation in the present situation is novel because we are solving vacuum equations and the only charges in the system are due to the $W^{ \pm}$fields.

The value of electromagnetic helicity $(\approx 2.5)$ is much less than the value estimated $(\approx 200)$ in Refs. $[5,6$. The estimate in [6] assumed a certain decay channel for linked loops of Z-string and it is likely that the electromagnetic helicity is a function of the precise instability through which the sphaleron decays. A more rigorous estimate of the final helicity needs to be done statistically, taking into account the conditions at the epoch of baryogenesis.

Once electromagnetic field helicity is produced, it will evolve, and eventually get frozen-in in the highly conducting ambient plasma. This is the scenario envisaged in Ref. [6] and, under certain assumptions about the inverse cascade, leads to the estimate that the cosmic magnetic field is $\sim 10^{-13} \mathrm{G}$ at recombination and coherent on a comoving scale of $\sim 0.1 \mathrm{pc}$. We now argue that this is really an underestimate of the magnetic field strength and typically we can expect a much higher field strength.

The essential point is that every baryon number violating reaction goes via the sphaleron and produces magnetic fields, whereas the estimate in [6] only accounts for the magnetic field produced due to the excess of baryons over antibaryons that we see today. To make this clearer, suppose that sphalerons decay in some volume to produce $N_{b}$ baryons while others decay to produce $\bar{N}_{b}=N_{b}-\epsilon$ antibaryons, where $\epsilon$ is entirely due to fundamental CP violation. Magnetic fields will be produced in each one of these $N_{b}+\bar{N}_{b}=2 N_{b}-\epsilon$ sphaleron decays. The baryon

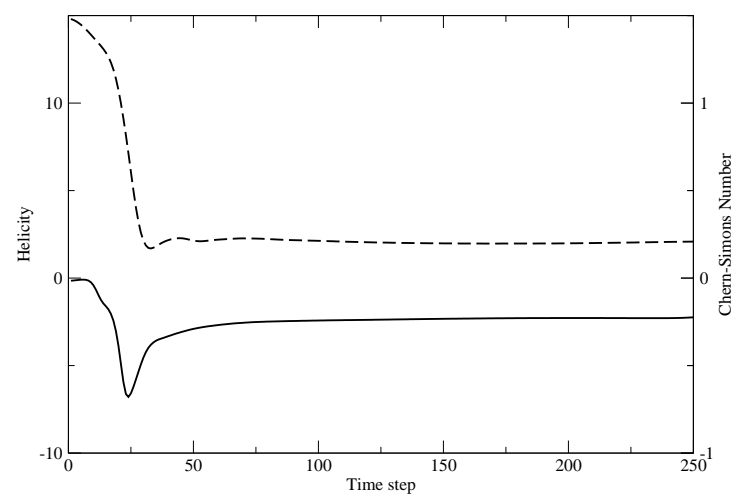

FIG. 1: Time evolution of Chern-Simons number (dashed curve) and electromagnetic helicity (solid curve). The sign of the Chern-Simons number has been flipped for greater clarity.

excess, however, is due to $\mathrm{CP}$ violation and is given by $N_{b}-\bar{N}_{b}=\epsilon$ sphaleron decays. The magnetic field produced by just these excess number of reactions is much smaller. However, just as the baryons and antibaryons can annihilate, it is likely that some of the magnetic fields produced due to baryon and antibaryon production will also annihilate. It is hard to estimate exactly how much magnetic field survives, but the estimate in [6] is the minimum value of the magnetic field. This value is protected by helicity conservation.

We will now obtain another estimate with less restrictive, and more realistic, assumptions about the evolution of the magnetic fields. Let us suppose that the magnetic fields due to baryon and antibaryon production do not completely annihilate, leading to a magnetic field enhancement by a factor $r$. Then

$$
B \sim 10^{-13} r G
$$

Successful big bang nucleosynthesis (BBN) constrains the energy density in the magnetic field so that $B\left(t_{\mathrm{rec}}\right)<1$ G. We choose the BBN bound as opposed to other bounds since BBN places strong constraints on magnetic fields with small coherence scale e.g. see 24]. (Other constraints due to gravitational wave production may apply to the non-helical component of the magnetic field [25].) This implies that

$$
r<10^{13}
$$

Now to connect to particle physics, we realize that $r$ depends on the total number of sphaleron processes, which are $N_{b}+\bar{N}_{b}$ in number. The estimate in [6] of $10^{-13}$ $\mathrm{G}$ at recombination assumes that the magnetic field is proportional only to the net baryon number $N_{b}-\bar{N}_{b}$. Assuming that the magnetic field strength gets reduced 
due to annihilations in some stochastic way, we expect

$$
r \sim\left(\frac{N_{b}+\bar{N}_{b}}{N_{b}-\bar{N}_{b}}\right)^{\gamma}
$$

where $\gamma$ is an undetermined exponent which is 1 for no annihilation and 1/2 for Brownian evolution of the magnetic field strength.

The baryon excess is purely due to $\mathrm{CP}$ violation in the particle physics responsible for baryogenesis. For example, in the case of the electroweak model [26],

$$
\frac{N_{b}+\bar{N}_{b}}{N_{b}-\bar{N}_{b}} \sim 10^{20}
$$

leading to $10^{-3} \mathrm{G}$ at recombination for the stochastic case $(\gamma=1 / 2)$ and $10^{7} \mathrm{G}$ for the no annihilation case $(\gamma=1)$. The latter estimate clearly violates the BBN bound in Eq. (18), while the former leads to $10^{-9} \mathrm{G}$ today though on short coherence scales (0.1 pc).

The argument above is intended to show that it may be possible to derive important model-independent constraints on particle physics from limits on cosmic magnetic fields. Further, if cosmic magnetic fields are observed, they can be used to derive detailed information about processes at baryogenesis and hence about high energy particle physics and $\mathrm{CP}$ violation.

We are grateful to Jose Blanco-Pillado, Juan GarcíaBellido, Margarita García, Noah Graham, Biagio Lucini and Jon Urrestilla for discussions. TV thanks the Netherlands Organization for Scientific Research (NWO) for a visitor's grant, and the Lorentz Institute of Leiden University, and ICTP (Trieste) for hospitality. This work was supported in part by the U.S. Department of Energy and NASA at Case Western Reserve University, and by NWO under the VICI programme.

[1] M. S. Turner and L. M. Widrow, Phys. Rev. D 37, 2743 (1988).

[2] T. Vachaspati, Phys. Lett. B 265, 258 (1991).

[3] B. Ratra, Astrophys. J. 391, L1 (1992).
[4] T. Vachaspati, arXiv:hep-ph/9405286

[5] J. M. Cornwall, Phys. Rev. D 56, 6146 (1997) arXiv:hep-th/9704022.

[6] T. Vachaspati, Phys. Rev. Lett. 87, 251302 (2001) arXiv:astro-ph/0101261.

[7] Diaz-Gil, A. Garcia-Bellido, J., Perez, M.G. \& GonzalezArroyo, A. 2007 Magnetic field production during preheating at the electroweak scale. arXiv:0712.4263 [hep$\mathrm{ph}$.

[8] R. Jackiw and S. Y. Pi, Phys. Rev. D 61, 105015 (2000) arXiv:hep-th/9911072.

[9] N. S. Manton, Phys. Rev. D 28, 2019 (1983).

[10] F. R. Klinkhamer and N. S. Manton, Phys. Rev. D 30, 2212 (1984).

[11] T. Vachaspati, Phys. Rev. Lett. 68, 1977 (1992) [Erratum-ibid. 69, 216 (1992)].

[12] T. Vachaspati and G.B. Field, Phys. Rev. Lett. 73, 373 (1994) arXiv:hep-ph/9401220.

[13] M. Hindmarsh and M. James, Phys. Rev. D 49, 6109 (1994) arXiv:hep-ph/9307205.

[14] J. Garriga and T. Vachaspati, Nucl. Phys. B 438, 161 (1995) arXiv:hep-ph/9411375.

[15] A. Achucarro and T. Vachaspati, Phys. Rept. 327, 347 (2000) [Phys. Rept. 327, 427 (2000)] arXiv:hep-ph/9904229.

[16] Y. Nambu, Nucl. Phys. B 130, 505 (1977).

[17] J. Kunz, B. Kleihaus and Y. Brihaye, Phys. Rev. D 46, 3587 (1992).

[18] J. Ambjorn, T. Askgaard, H. Porter and M. E. Shaposhnikov, Nucl. Phys. B 353, 346 (1991).

[19] G. D. Moore, Nucl. Phys. B 480, 689 (1996) arXiv:hep-lat/9605001.

[20] A. Tranberg and J. Smit, JHEP 0311, 016 (2003) arXiv:hep-ph/0310342.

[21] J. Garcia-Bellido, M. Garcia-Perez and A. GonzalezArroyo, Phys. Rev. D 69, $023504 \quad$ (2004) arXiv:hep-ph/0304285.

[22] N. Graham, Phys. Rev. Lett. 98, 101801 (2007) arXiv:hep-th/0610267.

[23] K. D. Olum and J. J. Blanco-Pillado, Phys. Rev. Lett. 84, 4288 (2000) arXiv:astro-ph/9910354.

[24] P. J. Kernan, G. D. Starkman and T. Vachaspati, Phys. Rev. D 54, 7207 (1996) arXiv:astro-ph/9509126.

[25] C. Caprini and R. Durrer, Phys. Rev. D 65, 023517 (2002) arXiv:astro-ph/0106244.

[26] A. Riotto and M. Trodden, Ann. Rev. Nucl. Part. Sci. 49, 35 (1999) arXiv:hep-ph/9901362. 